\title{
Fin de vie aux urgences : une mission incontournable en quête d'améliorations
}

\section{End-of-Life in the Emergency Department: an Unavoidable Mission in Need of Improvements}

\author{
P. Le Conte $\cdot$ E. Batard $\cdot$ G. Potel \\ (C) SFMU et Lavoisier SAS 2018
}

Depuis déjà une quinzaine d'années, $60 \%$ des décès en France ont lieu à l'hôpital, dont 10 à $16 \%$ aux urgences [1], et rien ne laisse prévoir un changement dans cette tendance. Dans une étude prospective ayant inclus 2420 patients décédés, $80 \%$ des décès aux urgences survenaient après une décision de limitation ou d'arrêt des thérapeutiques (LAT) [2]. Les principales caractéristiques de ces patients sont connues ; il s'agit d'une population âgée, voire très âgée, avec de lourds antécédents médicaux, une dépendance marquée [2], qui sont admis aux urgences pour les derniers moments de leur vie. Certes, il serait souhaitable de développer des alternatives, d'améliorer les soins palliatifs en EHPAD, d'accroître les directives anticipées, d'impliquer les médecins traitants, mais la réalité est là : il est plus que probable qu'un nombre croissant de patients comme ceux décrits ci-dessus vont continuer à être admis aux urgences, et nos services doivent s'adapter pour les prendre en charge dans les meilleures conditions possible [3].

Dans le même temps, une profonde évolution sociétale sur la fin de vie s'est développée avec la primauté de la volonté des patients, le refus de l'obstination déraisonnable et le droit aux soins palliatifs. Elle s'est traduite par l'adoption de lois (Leonetti puis Claeys-Leonetti) ainsi que par la publication de plusieurs recommandations de sociétés savantes, dont la dernière commune à la Société française de médecine d'urgence (SFMU) et à la Société de réanimation de langue française (SRLF) [4].

Aux urgences, les patients et les situations cliniques de prise de décision sont profondément différents de ce qui se passe dans les services de réanimation, il ne faut donc pas s'étonner que les délais entre l'admission, la décision de

P. Le Conte $(\bowtie) \cdot$ E. Batard $\cdot$ G. Potel

Service des urgences, CHU de Nantes,

9, quai Moncousu, F-44093 Nantes, France

e-mail : philippe.leconte@chu-nantes.fr

Université de Nantes, faculté de médecine,

1 , rue Gaston-Veil, BP 53508,

F-44035 Nantes cedex 01, France
LAT et le décès soient particulièrement brefs [2]. En réanimation, ces décisions se prennent presque toujours en cas d'échec thérapeutique malgré une stratégie bien conduite ou lorsque le patient refuse un nouveau traitement. Cela se déroule après une phase à visée curative permettant de nouer des relations avec les proches et de prendre, in fine, une décision collégiale. À l'inverse, aux urgences, il s'agit le plus souvent d'un patient inconnu des personnels du service, admis pour les dernières heures de sa vie, dans une situation clairement au-dessus de toute ressource thérapeutique.

Deux travaux rétrospectifs publiés dans ce numéro ont exploré les décès et les décisions de LAT dans deux hôpitaux différents $[5,6]$ et apportent des résultats très concordants. Les populations sont comparables entre elles et similaires à celle décrite ci-dessus. Le premier travail décrivant tous les patients décédés [5] retrouvait une proportion de LAT de $80 \%$ également similaire à la littérature [2]. Notons qu'aucune directive anticipée n'était retrouvée dans ces deux études, qu'une personne de confiance n'était désignée que chez huit patients sur 95 dans l'une et aucune dans la seconde. Cela témoigne de la mauvaise adhérence ou connaissance de la population de ces notions pourtant indispensables à l'expression de la volonté du patient.

Plusieurs évolutions peuvent cependant être notées depuis le travail réalisé en 2006 [2] alors que les caractéristiques des populations sont comparables : un moindre recours à la réanimation d'attente (30 et 15 versus $73 \%$ ), une amélioration de la traçabilité (74 et 86 versus $17 \%$ ), une augmentation des soins palliatifs prodigués (70 versus $56 \%$ ) $[5,6]$. Cela témoigne de l'amélioration de la prise en charge de ces patients grâce à une meilleure connaissance de ces situations par l'ensemble des équipes soignantes.

Néanmoins, il existe des pistes d'amélioration de prise en charge à partir de ces deux travaux et d'autres données de la littérature :

- l'une des études retrouvait une collégialité de $44 \%$ et l'autre de $80 \%$ [5,6]. La collégialité est nécessaire : une telle décision se doit d'être partagée et ne devrait pas 
reposer uniquement sur le médecin urgentiste. La récente recommandation commune propose des modalités pratiques en fonction du lieu et de l'horaire de cette prise de décision [4];

- les personnels soignants n'étaient pratiquement jamais impliqués dans la décision alors que leur présence est requise par les recommandations. Leur rôle est majeur, car ils apportent une autre vision que celle du corps médical. Une modification des procédures mérite d'être mise en place ;

- la famille n'a pu être contactée dans $25 \%$ des cas dans une étude et dans $32 \%$ dans l'autre [5,6]. Il s'agit là d'une situation regrettable, des mesures correctrices devraient être envisagées et les coordonnées familiales devant faire l'objet d'un enregistrement au moment de l'admission ;

- la traçabilité de la décision était de 74 et $86 \%$ [5,6]. Elle est pourtant indispensable à la continuité des soins entre l'accueil ou la SAUV et l'UHCD par exemple ;

- des soins palliatifs ont été prodigués dans près de $70 \%$ des cas dans les deux études essentiellement sous forme de perfusion de morphine. Ils ne se limitent pas à cette administration et peuvent inclure des soins de confort, un soutien moral du patient et/ou de ses proches. La formation à ces techniques des équipes soignantes dans leur ensemble est probablement nécessaire.

Le chemin parcouru dans l'amélioration de la prise en charge de ces patients est indéniable et mérite d'être mis en exergue. Des progrès restent cependant nécessaires. Certes, des procédures informatiques et/ou des protocoles d'aide à la décision et à la prescription mériteraient d'être évalués. Mais rien ne remplacera les efforts de formation ni les espaces de dialogue organisés au sein d'équipes médicosoignantes stables et confiantes.

\section{Références}

1. Lalande F, Veber O (2009) La mort à l'hôpital. Inspection générale des affaires sociales. http://www.ladocumentationfrancaise.fr/var/ storage/rapports-publics/104000037.pdf (Dernier accès le 30 mai 2018)

2. Le Conte P, Riochet D, Batard E, et al (2010) Death in emergency departments: a multicenter cross-sectional survey with analysis of withholding and withdrawing life support. Intensive Care Med 36:765-72

3. Kompanje EJO (2010) The worst is yet to come. Many elderly patients with chronic terminal illnesses will eventually die in the emergency department. Intensive Care Med 36:732-4

4. Reignier J, Boulain T, Carpentier F, et al (2018) Limitations et arrêts des traitements de suppléance vitale chez l'adulte dans le contexte de 1'urgence. Ann Fr Med Urgence 8:246-51

5. Berard L, Zeller J, Schaeffer M (2018) Décès et limitations thérapeutiques aux urgences : étude rétrospective des pratiques dans un centre hospitalier français pendant quatre ans. Ann Fr Med Urgence 8:223-30

6. Prabakar V, Ayllon-Milla S, Damas-Perrichet C, et al (2018) Évaluation des prises de décisions de limitations et arrêt des thérapeutiques dans un service d'accueil des urgences. Ann Fr Med Urgence 8:217-22 